\title{
Editorial of special section on Hybrid Information Security Technologies: part II
}

\author{
Sangsoo Yeo · Shiuh-Jeng Wang
}

Published online: 27 August 2014

(C) Springer Science+Business Media New York 2014

Recently, the widespread use of Grid and Cloud computing technologies for future generation computer systems is demonstrated by the huge amount of projects served by various hybrid information technologies. The hybrid information technology computing often requires the availability of a massive number of computers for performing large scale experiments. Traditionally, these needs have been addressed using highperformance computing and cloud computing solutions with clusters and super computers. In the future generation computing world, hybrid information technologies will play a very important role in convergence of high performance, cloud computing, communication, and all other computational sciences and application. In this special section, we solicit original work in all areas of Hybrid Information Security Technologies and this is first part of special section. In particular, we seek original work on identifying new research and development challenges, developing new techniques and advanced information technology solutions in Hybrid Information Technologies in High Performance and Cloud Computing for future computing systems, innovative information and communication technologies and applications. In a thorough peerreviewed process, eight manuscripts were selected for first review. The manuscripts were finally selected for this Special Section after the first and second review processes. Each manuscript selected was blindly reviewed by at least three reviewers consisting of guest editors and external reviewers.

\footnotetext{
S. Yeo $(\varangle)$

Mokwon University, Seo-gu, Daejeon, Korea

e-mail: ssyeo2010@gmail.com; sangsooyeo@gmail.com

S.-J. Wang

Department of Information Management,

Central Police University, Taoyuan 333, Taiwan

e-mail: sjwang@mail.cpu.edu.tw
} 
Finally, our thanks go to all editorial staffs for their valuable support throughout the preparation and publication of this Special Section. We would like to thank all authors for their contributions to this special issue and thank the referees who have critically evaluated the papers within the short stipulated time. 